\title{
Les économies de la Caraïbe face à l'ALENA
}

Le cas de Porto Rico

Karen L. Orengo-Serra

\section{OpenEdition}

Journals

Édition électronique

URL : http://journals.openedition.org/plc/747

DOI : $10.4000 /$ plc. 747

ISSN : 2117-5209

Éditeur

L'Harmattan

Édition imprimée

Date de publication : 1 janvier 1997

ISSN : 1279-8657

Référence électronique

Karen L. Orengo-Serra, "Les économies de la Caraïbe face à I'ALENA », Pouvoirs dans la Caraïbe [En ligne], Spécial | 1997, mis en ligne le 16 mars 2011, consulté le 10 décembre 2020. URL : http:// journals.openedition.org/plc/747 ; DOI : https://doi.org/10.4000/plc.747

Ce document a été généré automatiquement le 10 décembre 2020.

(c) Pouvoirs dans la Caraïbe 


\section{Les économies de la Caraïbe face à l'ALENA}

Le cas de Porto Rico

Karen L. Orengo-Serra

\section{Introduction}

1 Depuis le milieu des années 80 et le début des années 90 , on constate au niveau international un déplacement des centres de gravité économiques à cause de nouveaux accords commerciaux et de la libération des marchés. Ces politiques lancent à la communauté internationale des défis au niveau du développement économique.

2 En Amérique, l'Accord de libre échange nord-américain (ALENA) entre les Etats-Unis, le Mexique et le Canada, et l'expansion du commerce intra-zone sur toute la planète, défient ainsi les petites économies de la Caraïbe, notamment celle de Porto Rico et leur imposent de nouvelles contraintes.

3 C'est au cours des années 90, à la suite du lancement de l'Initiative pour les Amériques du Président nord-américain, George Bush, que les nouvelles tentatives d'intégration sousrégionales mises en place se sont accélérées. A partir de cette période, les conséquences de l'ALENA sur les économies de la Caraïbe ont pris de l'importance dans l'agenda politique des dirigeants caribéens. Le discours politico-économique a débouché sur une nouvelle phase où le processus d'intégration de la région et son incorporation à l'espace élargi centré sur les Etats-Unis (l'ALENA), s'est imposée afin de ne pas rester en marge des mutations de l'économie mondiale.

Dans la Caraïbe, au cours des années 1980, Porto Rico commence à prendre conscience de ces transformations et du réalignement des divers pôles économiques mondiaux. En effet, à la suite du lancement de la Caribbean Bassin Initiative (CBI), Porto Rico intensifie de façon significative ses rapports avec les pays voisins de la Caraïbe et acquiert ainsi un rôle clé dans l'articulation des politiques de développement politico-économique dans le bassin de la Caraïbe. A partir de cette époque, Porto Rico joue de plus en plus le rôle de plaque 
tournante, de pivot du dispositif de la politique nord-américaine dans la nouvelle dynamique de restructuration de la région.

Une évolution des rapports entre les pays de la Caraïbe et les Etats-Unis est inévitable. Les pays de la région attendent encore de voir quelle direction de la politique nordaméricaine et quelles en seront les conséquences sur les relations Caraïbes Etats-Unis, particulièrement dans le cadre de l'ALENA. Quant aux Etats-Unis, il semble qu'ils n'aient pas encore développé une stratégie systématique pour la Caraïbe en dehors de l'ALENA. Emerge seulement une vague notion selon laquelle les dispositions de l'ALENA seraient étendues au reste de la Caraïbe et de l'Amérique Latine'.

Une nouvelle dialectique d'intégration des pays de la Caraïbe apparaît où l'ALENA fait partie des grands projets d'association régionale au niveau de l'hémisphère centré sur les Etats-Unis et de la restructuration économique internationale en cours. Cela représente un défi pour les petites économies de la Caraïbe car ces pays doivent restructurer leurs modes de production industrielle afin de s'adapter aux nouvelles exigences des marchés globaux ${ }^{2}$. Selon Watson, les pays de la Caraïbe ne savent pas exactement comment atteindre cet objectif et cela pose problème. L'un des éléments les plus déstabilisants pour les économies caribéennes est principalement, l'adhésion du Mexique à l'ALENA ; tandis que l'autre est constitué par la mise en place d'une nouvelle forme de dépendance de la Caraïbe vis à vis des politiques exogènes gérée principalement par des organismes internationaux (comme le Fond Monétaire International et la Banque mondiale).

7 Cet article propose d'analyser la position des petites économies de la Caraïbe face à l' ALENA et aux nouvelles tendances du processus international de production en prenant le cas de Porto Rico. Dans un premier temps, nous présenterons le cadre historique des stratégies de développement économique de la Caraïbe et les évolutions subies par cellesci en réponse aux transformations du nouvel ordre économique international. Ensuite, nous verrons comment la nouvelle conjoncture économique mondiale influe sur les économies de la Caraïbe confrontées au grand marché de l'ALENA. Enfin, nous montrerons que les pays de la région ont peut-être déjà commencé à tracer une voie d'accès à l'ALENA pour s'intégrer ainsi au schéma de l'économie mondiale du XXI ${ }^{\mathrm{e}}$ siècle.

\section{Les stratégies de développement économique de Porto Rico et des pays de la Caraïbe}

8 Dans les premières décennies du $\mathrm{XX}^{\mathrm{e}}$ siècle, l'économie des pays de la Caraïbe était organisée autour du secteur agricole. C'était une économie de plantation dont la production était destinée aux marchés métropolitains au détriment du marché domestique. Ce n'est que plus tard que les mutations de l'économie internationale lors de la Seconde guerre mondiale ont engendré un contexte favorable au développement d'une pensée économique caribéenne. Avec cette génération d'intellectuels conscients des contraintes économiques des pays de la région les gouvernements de la zone vont être poussés, à partir des années 50 à adopter des politiques tournées vers le développement et la transformation structurelle de ces économies.

9 Inspirée par les travaux d'Arthur Lewis, la première stratégie avait pour objectif le développement du secteur industriel par le biais du processus de substitution des importations. Il s'agissait de l'industrialisation sur invitation, destinée à attirer le capital étranger. A Porto Rico, cette stratégie porte le nom d'Opération Bootsrap. Une main- 
d'œuvre bon marché et une exonération fiscale sur les revenus obtenus par les entreprises installées dans les pays d'accueil, en sont les principaux avantages offerts.

Cette stratégie n'a pas réussi cependant à impulser un développement soutenu et une croissance économique permettant de faire face aux problèmes sociaux de la région. Les entreprises établies dans ces pays n'avaient que très peu d'influence et de retombées sur l'économie domestique de ces pays. Elles constituaient des enclaves d'exportation très peu liées aux autres industries locales des petites économies de la Caraïbe. Il s'agissait en règle générale, de filiales des entreprises intégrées verticalement dont le siège se trouvait à l'étranger, principalement dans la métropole. Les filiales localisées dans les pays de la Caraïbe avaient une fonction d'usine d'assemblage ou de production de type maquiladora, requérant un usage intensif en capital et important en matières premières ou partiellement industrielles, et réexportant le produit final vers les marchés métropolitains.

11 Les résultats plutôt décevants de cette stratégie se traduisirent par le début d'une vague d'activisme politique et social dans la région et le développement accéléré du rôle plus actif de l'Etat dans la politique économique des pays de la zone. L'application de l'interventionnisme de l'Etat se manifesta, dès le départ, et le plus souvent par des nationalisations d'entreprises de services publics et parfois des nationalisations dont l'objectif était la sauvegarde des emplois existants. Ainsi, on constate, d'abord en Guyana, la mise en place de la coopérative socialiste en 1970, puis à Trinidad \& Tobago, en 1972, et en Jamaïque, en 1974. Les autres pays de la région continuèrent à mettre l'accent sur le rôle primordial des capitaux étrangers alors que l'Etat allait progressivement intervenir dans les activités économiques.

Plus tard les tentatives d'intégration régionale se sont renforcées et ont joué un rôle important dans les stratégies de développement menées par les dirigeants des économies insulaires. Il faut souligner à cet égard, que l'idée de coopération régionale a été toujours présente dans l'esprit des Caribéens. Car ce processus permettait le développement des économies d'échelle, l'élargissement du marché des différents pays concernés et la sécurité d'une distribution optimale des ressources, déjà rares, de la région.

En effet, suite à l'échec de la Fédération des West-Indies

(1958-62), qui avait été créée par la Grande Bretagne dans le but d'organiser un ensemble régional lié à la métropole, plusieurs tentatives de coopération régionale ont été menées. Cette volonté d'intégration et de coopération économique s'est traduite par l'instrumentalisation de nouvelles mesures institutionnelles dont le but était double: d'une part, favoriser la formation d'un marché régional pour pallier l'étroitesse des marchés locaux; d'autre part, établir une organisation capable de négocier avec les partenaires de la région ${ }^{3}$.

Dans les années 1960-70, une zone de libre échange, CARIFTA (Caribbean Free Trade Association) fut organisée dans les pays de la Caraïbe anglophone, relayée quelques années après par la CARICOM, dont le but était la constitution d'un marché commun".

La crise économique mondiale qui éclate à la fin des années soixante et les mutations qu'elle entraîne au niveau de l'économie mondiale, font que les tentatives d'intégration au cours des années 80 prennent un autre aspect: la libéralisation des échanges, l'ouverture des économies et la redéfinition du rôle de l'Etat. Elles ont pour but de restructurer les économies des pays membres de la CARICOM et de redéfinir la spécialisation de ces pays dans la compétition internationale. En effet, on assiste à une 
restructuration économique centrée sur la spécialisation et la production intégrée à partir de Porto Rico. La CBI doit prescrire les normes de production et de commerce entre ces pays et les Etats-Unis. Néanmoins, dans l'ensemble de ses dispositions, la CBI n'a pas atteint les résultats escomptés. Seulement un nombre restreint de pays ont pu bénéficier de cette initiative surtout grâce aux fonds de laSection $936^{5}$.

La conjoncture économique internationale actuelle et les mutations qu'elle entraîne exigent des pays périphériques, notamment ceux de l'ensemble caribéen, un réaménagement structurel de leurs économies articulé sur un nouveau processus d'intégration, et de production prenant en compte des variables technologique et de spécialisation. Ce réaménagement, comme l'on verra plus loin, devient impératif face aux formations des blocs économiques, en particulier, le grand marché de l'ALENA.

\section{Le cas de Porto Rico}

Depuis les années 1950, le modèle de développement économique de Porto Rico a été caractérisé par un modèle d'accumulation du capital basé sur les investissements étrangers, surtout des investissements nord-américains.

18 A ses origines, le modèle portoricain connu comme l'Operation Bootstrap, prévoyait l'attraction des investisseurs nord-américains qui fabriqueraient des marchandises sur l'île pour le marché extérieur. L'exonération d'impôts sur les revenus était le pilier de la stratégie. Les avantages structurels qu'apportaient ce pays au marché nord-américain restent cependant, les clefs stimulantes de l'operation Bootstrap. Il s'agissait de faibles salaires ou d'une main-d'œuvre bon marché, du libre mouvement de produits entre les marchés de Porto Rico et les Etats-Unis, dû à la relation coloniale qui faisait de l'île une partie intégrante du système commercial interne américain, de l'exonération d'impôts par le gouvernement fédéral et celui de Porto Rico sur les revenus des filières localisées dans l'île, de la proximité géographique de ce pays avec les Etats-Unis et de l'infrastructure étatique sociale et réformiste qui renforçait la mise en vigueur du modèle d'industrialisation.

Le succès relatif du modèle portoricain tout au long des dernières décennies s'explique en partie, par la capacité du gouvernement de Porto Rico d'instrumentaliser des politiques pragmatiques capables de concilier les intérêts de la population, ceux de la métropole et également les intérêts de l'élite politique au pouvoir. Les différentes stratégies de restructuration du modèle mises en vigueur par les différents gouvernements locaux face aux crises témoignent de la façon dont le gouvernement portoricain a maitrisé les contraintes qui mettaient en danger l'Operation Bootstrap.

Pendant les dix premières années, différentes contraintes provoquèrent une restructuration du modèle entre 1960 et 1975. Il fut orienté vers l'importation de capitaux à travers l'industrie lourde, à savoir l'industrie pétrochimique ${ }^{6}$. En 1976, le modèle entra dans une deuxième phase de restructuration. Le nouvel axe du développement économique concernait les industries à haut contenu technologique favorisées par la Section 936. Avec cette législation, les entreprises localisées dans l'île étaient exonérées d'impôts fédéraux sur le bénéfice des activités industrielles et de services produits par l'entreprise sur le territoire, ainsi que sur les dividendes rapatriés vers la maison mère.

21 Contrairement à la Section 931, qui était la base de l'opération Bootstrap à l'origine, sous la Section 936 le gouvernement de Porto Rico pouvait prélever une toll-gate tax sur les 
profits rapatriés vers les Etats-Unis. La toll-gate tax était un impôt qui poussait les entreprises à investir une partie des bénéfices acquis en certificats de dépôts bancaires spéciaux. Si elles n'effectuaient pas l'investissement, les entreprises étaient dans l'obligation de payer la toll-gate tax sur leurs revenus rapatriés. Cet impôt permettait au gouvernement portoricain d'une part de faire des investissements et d'autre part, de transformer ce pays en un centre bancaire international.

A partir de cette époque, toutes les industries nord-américaines établies dans l'île eurent recours à la Section936, particulièrement les industries à haute technologie qui produisaient des produits à grande valeur ajoutée et des bénéfices élevés.

23 Jusqu'à aujourd'hui, la Section 936 a été le centre du développement économique de l'île. A cela s'ajoutent les transferts fédéraux comme les food-stamps, les bourses Pell Grants pour études supérieures, les subventions sur les services publics (l'électricité et l'eau), et plus récemment l'assurance maladie privée qui est attribuée aux populations démunies.

Dès le départ la Section 936 a été mise en question. Toutefois, la stratégie qui consistait à utiliser une partie des fonds de la Section 936 pour encourager le développement des économies de la Caraïbe dans le cadre de la CBI, a permis un renforcement et un élargissement du modèle à partir de 1986.

Cette stratégie consistait en une modification de la Section 936 , qui permettait le financement des entreprises joint-ventures dans les pays de la zone, autrement dit, l'intégration du processus productif d'un certain nombre des pays de la zone Caraïbe (les pays bénéficiaires de la $\mathrm{CBI}$ ). La réforme en question institua, à partir de ces exonérations, un fond annuel spécial de 100 millions de dollars US, le Qualified Possession Source Investment Income (QPSII), destiné à accorder des crédits à des taux privilégiés pour financer des twin-plants génératrices d'emplois à Porto Rico et dans les pays éligibles de la région.

26 Avec le concept des entreprises joint-ventures, la production plus intensive en maind'œuvre allait être réalisée dans un pays de la région, la production technologiquement plus avancée devant être terminée à Porto Rico. De là, le produit fini devait être exporté vers le marché nord-américain et d'autres marchés internationaux.

27 La participation de Porto Rico à la CBI à travers les Fonds 936, lui permettait de mettre en œuvre une politique américaine dans la Caraïbe en échange du maintien de la Section 936 ${ }^{8}$, qui le conduisait à une plus grande autonomie avec le consentement tacite ou explicite de Washington et à une plus grande insertion aux marchés de la région. Lors de cette modification de la Section 936, Porto Rico commence non seulement un véritable processus d'intégration de la région, mais aussi à devenir un leadership de la politique américaine vers la zone. Comme le souligne Aaron Ramos, Porto Rico «se convertit progressivement en élément pivot de la politique américaine dans la Caraïbe, pérennisant ainsi l'utilisation de la Section 936 du code fédéral qui permet de défiscaliser les bénéfices réalisés par les firmes américaines implantées dans les territoires extérieurs $»^{9}$.

$\mathrm{Au}$ fur et à mesure, les opérations de production complémentaire (twin plants) dans Porto Rico et les pays de la zone, vont placer Porto Rico dans une position prépondérante au sein du circuit mondial des capitaux nord-américains. En effet, avec l'accord au cours des années 90 sur l'extension de la CBI-II, Porto Rico accroît son rôle dans les affaires économiques de la zone. Le montant total des fonds assurant le financement des projets dans la Caraïbe s'élevait à 233,068 millions de dollars US en 1991, 183,013 millions en 1992 et 202,539 millions en 1993. 

secteurs économiques puissants (finances, services, manufacture de biens à haut contenu technologique), les constants débats autour de la Section 936 au sein du Congrès américain ont conduit récemment à son élimination. Plusieurs questions sont posées à travers cette décision. Il faut remettre en question les rapports de pouvoir entre les EtatsUnis et Porto Rico car la décision prise par le Congrès américain ne tient pas compte de l'autonomie apparente qu'avait acquise au fil du temps le gouvernement de Porto Rico ni de la fragilité de son pouvoir vis-à-vis du peuple et notamment de l'ensemble caribéen. Cette détermination remet également en question la politique suivie par les Etats-Unis dans la zone car l'absence des Fonds 936 limite le rôle de Porto Rico dans le processus d'intégration de la Caraïbe, mais aussi dans la mise en place de la politique américaine.

la prise de cette décision n'est-elle pas le début d'une évolution nécessaire des relations entre les Etats-Unis et Porto Rico dans le contexte de l'actuelle politique économique américaine? Cette décision ne représente-t-elle pas l'une des premières démarches à suivre permettant le passage du pays au grand marché de l'ALENA ? Cette détermination, n'est-elle pas le départ d'un nombre considérable de préférences et d'avantages en train de disparaître devant l'ALENA ? Ou bien, est-il possible que cette décision n'en soit qu'un élément?

Le Projet 30-A qui remplace la Section 936 n'a fait qu'allonger la période d'exonération fiscale à 10 ans dans le cas des entreprises déjà établies. Le problème qui se pose est qu'il n'y a pas encore de système semblable qui puisse attirer des investissements étrangers. Il semble que les élites politiques insulaires soient en train d'élaborer de nouvelles stratégies de développement à l'intérieur du Projet Young, qui prévoit la définition du statut portoricain dans un futur proche.

En effet, comme on le verra plus loin, les exigences de ce nouvel ordre économique dans le cadre de l'accession à l'ALENA des problèmes à Porto Rico et pour l'ensemble des pays de la Caraïbe. La restructuration du modèle de développement portoricain et celui de l'ensemble des pays de la Caraïbe sont impératifs. Mais, le processus d'intégration régionale pose la question de savoir si l'accession au marché de l'ALENA va être articulée par chaque pays de façon individuelle ou bien par l'ensemble des pays organisés, soit la CARICOM ou la CBI, soit par l'ancienne Association des Etats de la Caraïbe. Quelques pays de la Caraïbe comme la Jamaïque, Trinidad et Tobago ont déjà déposé leur candidature pour adhérer au grand marché de l'ALENA. Cela risque d'être une entrave pour le processus d'intégration économique de la région, toujours remis en cause ${ }^{10}$.

\section{La Caraïbe dans le cadre de l'ALENA}

L'histoire du développement économique des pays du bassin de la Caraïbe, montre que le marché nord-américain a toujours joué un rôle très important à l'intérieur des stratégies de développement, mises en oeuvre après la Seconde guerre mondiale dans la région. En effet, la première source de biens importés reste les Etats-Unis avec $39 \%$ des importations, alors que l'Europe représente $14 \%$, l'Amérique Latine $11 \%$ et l'ensemble de la CARICOM $9 \%$. En ce qui concerne les exportations, les Etats-Unis sont encore une fois la destination préférée avec $40 \%$ de biens exportés, suivi de l'Europe avec $18 \%$ et, en particulier la Grande Bretagne avec $11 \%$.

Pouvoirs dans la Caraïbe, Spécial | 1997 

potentiel pour leurs exportations de produits non traditionnels. Grâce aux dispositions de préférences commerciales sous le programme Système de préférences généralisé (SPG) et celui des Zones franches industrielles d'exportation (ZFIE), les exportations de produits non traditionnels de la CBI vers les Etats-Unis ont connu un accroissement de l'ordre de $64 \%$ des exportations totales destinées au marché nord-américain. Cela explique l'augmentation des exportations d'assemblage de vêtements de 1108 millions de dollars US en 1987 à 2440 millions en 1991, soit une croissance de 21,8\%. Le doublement de ces exportations s'explique aussi par la présence de nombreuses entreprises localisées en République Dominicaine sous le programme des ZFIE ${ }^{11}$. Concentrée principalement au Costa Rica, en Haïti, en Jamaïque et surtout en République Dominicaine, la part des exportations des ZFIE dans les exportations totales de ces pays est passée de 16,8\% en 1983 à 36,4 \% en 1987.

La nouvelle conjoncture de l'économie internationale ne semble pas être très favorable aux petites économies de la Caraïbe. Ces petits pays périphériques ne pourront plus compter sur les liens traditionnels qui demeurent entre eux et l'environnement industrialisé. La libéralisation de l'accès au marché nord-américain dû aux accords de l' ALENA, du GATT entre autres, engendre une concurrence plus intensive sur les marchés d'exportations des petites économies de la zone Caraïbe et réduit ses avantages face au Mexique et au Canada dans le cadre de l'ALENA. La tendance à la diminution des barrières douanières aura des implications sur les industries à forte main-d'œuvre, qui dans le cas portoricain rapportent $38 \%$ de l'emploi dans le secteur industriel.

En outre, adhérer à l'ALENA se traduit pour certains pays de la CARICOM, par l'abandon des accords privilégiés de Lomé dont ils bénéficient et qui sont beaucoup plus attrayants que ceux de l'ALENA. La Caraïbe présente des contraintes qui limitent leur adhésion à l' ALENA mais il y a aussi des variables qui favorisent leur insertion à l'économie de grands blocs.

37 A Porto Rico et dans la Caraïbe, le modèle de développement se traduit par la production industrielle de type maquiladora. L'industrie maquiladora dans la Caraïbe et au Mexique jouit des bénéfices tarifaires similaires dans le marché nord-américain, mais les producteurs caribéens profitent des meilleures préférences commerciales. En 1988, par exemple, les exportations d'assemblage de vêtements en provenance de la CBI vers les Etats-Unis ont bénéficié d'un tarif de $4,9 \%$ contre $8,6 \%$ pour les importations d'assemblage de vêtements en provenance du Mexique. En outre, en 1975, $75 \%$ de la valeur des exportations d'assemblage de vêtements en provenance de la CBI destinées au marché nord-américain était constituées de composants américains contre $55 \%$ des exportations similaires en provenance du Mexique ${ }^{12}$.

Les exportations non traditionnelles, surtout les textiles et les vêtements, représentent 2 milliards de dollars US, soit $25 \%$ des exportations annuelles des pays de la CBI, autrement dit, 90 à $95 \%$ des achats nord-américains. Cette activité industrielle rapporte 200000 emplois dans la région; 25000 emplois, soit $12 \%$ pour la Jamaïque. L'industrie textile et d'assemblage de vêtements est très importante pour les pays de la Caraïbe. Dans le cadre du GATT, les politiques protectionnistes qui soutiennent cette industrie, entrent dans une phase visant leur disparition. En effet, laUS International Trade Commission (USITC) soutient l'élargissement de l'ALENA aux pays membres de la CBI, lors de la suppression immédiate des tarifs préférentiels sur les importations sous le programme SPG (Programme 807A) et 
de la disparition progressive pendant la période de 6 ans des tarifs sur d'autres importations qui ne font pas partie du SPG.

Comme le précise Hilbourne Watson, une alternative à envisager pourrait être les politiques qui vont être adoptées par le US Textile Industry Complex. Elles visent le maintien du protectionnisme dans cette industrie et leurs activités industrielles d'outre-mer. Néanmoins, le but de l'ALENA dans le cadre de l'Entreprise pour les Amériques, est de créer un marché fondé sur la notion de réciprocité, dans le but de surmonter les limitations que représentent un système de préférences unilatéral comme celui de la CBI. Le problème est que la plupart des pays membres de la CBI et de la CARICOM ne remplissent également les conditions pas non plus d'adhésion à ce système.

Quoique le marché de la Caraïbe ait été très important pour les exportations nordaméricaines plus que pour les exportations caribéennes, les avantages comparatifs que possède le Mexique en termes de coût de production (main-d'œuvre bon marché, coût de transport moins élevé, et produits énergétiques à bon marché) risquent de mettre à l'écart les productions caribéennes. Lorsque s'y ajoutent l'abaissement et la suppression des barrières tarifaires et non tarifaires, la position des économies de la Caraïbe se fragilise.

Il est vrai que la Caraibe n'a jamais eu une tradition productrice de classe capitaliste industrielle moderne ni celle d'une technologie avancée. Toutefois, on constate dans la région depuis quelques années, une adaptation aux variables d'innovation technologiques, surtout à Porto Rico et dans quelques pays anglophones. Les principaux centres d'industries maquiladoras et joint-ventures ont inclus celles qui avaient une connaissance technologique élevée à Porto Rico, en République Dominicaine et en Jamaïque. En prenant l'exemple de Porto Rico, il faut souligner certains avantages comparatifs de ces industries dans la région qui au fur et à mesure pourront permettre son adhésion au grand marché de l'ALENA. Il s'agit plutôt des variables technologiques, du savoir-faire, et de l'efficacité de la spécialisation dans le secteur de services et le secteur de la manufacture de produits à haute technologie.

Porto Rico et quelques pays de la Caraïbe anglophone ont une main-d'œuvre hautement qualifiée. Le savoir-faire et la production de ce que l'on connaît comme les high-techs industries, telles que les industries électroniques et pharmaceutiques, offrent par conséquent des bénéfices non négligeables. En outre, la spécialisation dans le secteur des services a fait de la région une zone privilégiée en terme de développement des services touristiques avec des flux et des recettes touristiques supérieurs à ceux de l'ensemble des pays latino-américains.

En effet, entre les critères d'implantation des firmes multinationales (FMNs) à l'étranger, les bas salaires sont moins priviligiés qu'une main-d'œuvre qualifiée et flexible, qu'un environnement technologique évolué ou qu'une infrastructure de qualité.

La tendance du commerce international au cours des années 1990, se caractérise par une évolution remarquable dans la structure sectorielle des flux, à savoir la contribution décroissante des services financiers et l'immobilier, et, en revanche, la poussée des autres services marchands et des activités de haute technologie. On constate également, un déplacement vers les services de réseaux et vers le manufacturier à haute et à moyenne technologie ainsi que des conditions favorables qui avaient, jusqu'au début des années 1980, favorisé l'internationalisation des services financiers. 
Simultanément, on assiste à une transformation des activités financières (banque et assurances) avec, d'une part, une forte ouverture des marchés nationaux aux entreprises étrangères, et d'autre part, la formation de véritables multinationales de services. La part des firmes de services dans les emplois américains à l'étranger est, par exemple, passée de $9 \%$ en 1982 à plus de $14 \%$ en $1993^{13}$.

On observe également, une accélération marquée dans l'industrie des services de réseaux, de haute technologie, de distribution et de services non financiers (le tourisme et l'hôtellerie). La participation des firmes de services dans la création d'emplois des filières américaines à l'étranger est, par exemple, passée de $9 \%$ en 1982 à plus de $14 \%$ en 1993. Pour sa part, l'industrie de la manufacture traditionnelle, l'industrie pharmaceutique, l'agro-alimentaire et l'industrie des services financiers et assurances ont connu une croissance moyenne pendant cette période. Ces industries représentent $68 \%$ des stocks actuels d'Investissements Directs à l'Etranger dont $27 \%$ pour le secteur financier, $17 \%$ pour la chimie, la pétrochimie et la pharmacie, $9 \%$ pour les industries automobiles et d'équipement.

Depuis la fin des années 70, à Porto Rico, par exemple, les industries à haute technologie (pharmaceutiques, équipement, électroniques et non-électroniques, outils professionnels et scientifiques) ont connu une croissance. La participation de ce secteur au PIB, à l'emploi ainsi que dans les exportations portoricaines est très significative. Pendant l'année fiscale 1995-96, la part de ce secteur dans le PIB, l'emploi et les exportations a été respectivement de $41,8 \%, 17 \%$ et $99,3 \%{ }^{14}$. Cette année, la part de la manufacture en général dans le PIB a atteint plus de $40 \%$, malgré les effets négatifs de la concurrence mondiale sur ce secteur.

4 Dans le secteur de la haute technologie, par exemple, plusieurs filiales des FMNs installées à Porto Rico, ont déjà commencé une phase de restructuration et d'expansion des installations.

La filiale Ocular Science (avec le financement de la Government Development Bank for Puerto Rico a investi \$15,8 millions dans la restructuration de ces installations. En 1995, la maison mère, localisée aux Etats-Unis, prévoyait sa fermeture, mais le PDG et les employés mirent en place une campagne visant le maintien des opérations. Cela se traduisit par une forte augmentation de la production grâce à la mise en place de nouvelles techniques de production et à la réduction significative des coûts de production. En deux ans seulement, la production passa de 5000 à 80000 lentilles de contact par jour. L'emploi connut une hausse de 170 à 300 . Il se peut qu'avec cette restructuration, le nombre d'emplois arrive à 400 et la production à 125000 lentilles de contact par jour ${ }^{15}$.

5 Aujourd'hui, l'Ocular Science de Porto Rico est la filière de production la plus importante de l'American Hydron Corp., localisée à San Francisco.

51 L'implantation de FMNs à Porto Rico se fait pour plusieurs raisons dont, en particulier, selon les PDG de certaines entreprises installées, la garantie d'une main d'œuvre productive, d'infrastructures de qualité et de conditions favorables aux investissements.

Le secteur des services a connu aussi une croissance, en particulier, pour l'exportation : le commerce, les sous-secteurs des services (services personnels, commerciaux, de récréation, médicaux, le tourisme, etc.) et le gouvernement. En 1994-95, ceux-ci ont rapporté $75 \%$ de l'emploi total et $39,2 \%$ du PIB. 
Entre 1963-64 et 1992-93, par exemple, la participation du secteur des services à l'emploi fut plus importante que celle de la manufacture. L'emploi dans l'industrie des services s'accrût de $3 \%$ tandis que dans la manufacture il augmentait de 1,9\%.

Dans le secteur des services, le tourisme, occupe une place très privilégiée dans la plupart des pays de la Caraïbe. Le rôle du tourisme international est issu d'une forte spécialisation de l'économie et des échanges extérieurs de ces pays. Les pays de la Caraibe ont, par exemple, un nombre d'arrivées touristiques supérieur (12,4 millions de touristes) à celui des pays d'Amérique du Sud (11,8 millions de touristes). Pour une population de 450 millions contre 30 pour les pays de la zone Caraibe ${ }^{16}$.

Porto Rico est la première destination touristique dans la Caraïbe à la fois en nombre de touristes et en recettes. En 1992, elle représentait environ $25 \%$ des touristes dans les Caraïbes soit 2,6 millions de personnes.

Il faut signaler que le développement touristique à Porto Rico vient largement de l'importance des infrastructures d'accueil et d'hébergement, mais aussi des facilités dues au transport aérien. L'aéroport de San Juan, permet à l'île d'être reliée à la fois à la majeure partie des grands aéroports américains et aux aéroports de l'ensemble des pays de la Caraïbe. Il joue ainsi un rôle central dans le tourisme caribéen.

Le gouvernement de Porto Rico, à travers la mise en vigueur de l'Acte pour le développement touristique de 1993 (TDA), a permis l'accroissement des investissements étrangers, par exemple, dans l'hôtellerie, avec l'implantation d'une filière de la chaîne des hôtels RitzCarlton, Inc., le Hampton Inn Hotel, l'hôtel El Convento, entre autres.

58 En outre, le développement touristique a eu un impact très favorable sur l'industrie de la construction. En 1996, l'industrie de la construction a connu une augmentation de 26,4 \%, (le plus haut pourcentage de croissance depuis 1988). L'investissement privé dans ce secteur a crû de $20,3 \%{ }^{17}$.

59 Entre 1973 et 1992, les courants d'échanges les plus dynamiques sur le plan mondial qui concernaient le commerce de l'Asie : d'abord le commerce intra-zone, puis le commerce avec l'Amérique du Nord et, enfin, celui avec l'Europe occidentale ${ }^{18}$. Pour les années à venir, il faudra analyser les échanges internationaux selon un système hiérarchisé de zones ou de régions qui comprend un pays ou un groupe de pays leaders, une sous-zone en développement fortement reliée au leader par son commerce, et des initiatives de coopération intra-régionale. Dans cette perspective les pays de la zone Caraibe ont désormais intérêt à se rapprocher de plus en plus de ce système hiérarchisé d'échanges commerciaux et financiers. Cette logique exige un changement de la politique sur le plan de la production, de la technologie et des rapports diplomatiques avec ces acteurs.

Dans la Caraibe et notamment à Porto Rico, la manufacture et les services jouissent d'un avantage non négligeable dans leurs échanges internationaux. Si l'on analyse les évolutions structurelles par produits du commerce international au début des années 90 , on constate la suprématie des produits manufacturés. Ils ont contribué à développer un type de commerce appelé intra-branche ou intra-industriel. Ce type de commerce fait appel alors à des spécialisations entre pays, beaucoup plus fines que celles qui consistent à exporter des machines et à importer des produits alimentaires.

Les échanges intra-branches entre Porto Rico et la République Dominicaine, par exemple, constituent un exemple non négligeable dans le processus d'intégration régionale. Il s'agit des échanges intra-industriel des produits décomposables déterminés par l'innovation et l'écart technologique. La décomposition des étapes de production des 
biens permet un commerce international d'exportation et de réimportation. Il comprend à l'assemblage à l'étranger, la fabrication de certaines pièces, le stockage, etc.; selon les avantages comparatifs de type dotations de facteurs comme une main-d'œuvre bon marché dans le cas de la République Dominicaine, un travail qualifié et une avance technologique dans le cas de Porto Rico.

Le commerce international a tendance à mettre l'accent sur la spécialisation. Le système de production d'assemblage type maquiladora dans la Caraïbe ne pourra continuer à être à la base des politiques de développement économique. Même, les entreprises joint-ventures ne jouissent pas d'une stabilité à long terme. Les variables de spécialisation et d'innovation progressive représentent des avantages comparatifs vis-à-vis du Mexique. Dans le cadre de l'ALENA, par exemple, celles-ci peuvent contribuer au développement du système hiérarchisé conçu. Dans ce contexte, Porto Rico et quelques pays de la Caraïbe anglophone se trouvent dans une position plus avantageuse que le Mexique.

Depuis quelques années Porto Rico essaie d'instrumentaliser une nouvelle structure de développement en matière de productivité, de recherche et de développement technologique. En fait, la productivité et la main-d'œuvre qualifiée mais aussi l'environnement favorable aux investissements jouent un rôle déterminant dans la concurrence mondiale pour attirer des capitaux étrangers. Dans ce contexte, il faudra remettre en cause la politique économique axée sur les incitations fiscales afin d'attirer les investissements étrangers.

64 A Porto Rico, par exemple, et dans le cadre de la CBI, les incitations fiscales sous la Section 30-A ne permettent pas de penser qu'il sera possible d'attirer de nouveaux investissements. En plus, les FMNs installées regardent avec incertitude la Section 30-A ; pour les joint-ventures l'avenir est incertain. En ce qui concerne les FMNs high-techs, particulièrement, les industries pharmaceutiques, on peut se demander si une maind'œuvre qualifiée, un haut niveau d'innovation technologique, de recherche et de développement va être assez importants pour empêcher les FMNs de délocaliser vers d'autres marchés. Si on ne développe pas un processus productif centré sur la spécialisation et l'innovation continue, semblable à celui des Nouveaux pays industriels ( $\mathrm{NPI}$ ), il ne serait pas étonnant d'assister à l'explosion du nombre de déplacements des entreprises vers d'autres marchés; car les incitations fiscales vont disparaitre aussi bien que la main-d'œuvre bon marché face aux bas salaires que représentent les marchés chinois et russes.

65 A cela s'ajoutent le poids de la dette et de la balance de paiements dans certains pays de la région. En outre, la dégradation continue des termes de l'échange des produits caribéens traditionnels dûe à la chute des cours de ces produits n'augure pas non d'un avenir favorable plus pour les pays de la zone.

Il me semble que chaque pays de la Caraïbe doit envisager l'adhésion au marché de l' ALENA selon un système de production hiérarchisé axé sur les rapports intra-régionaux et les nouveaux déterminants du commerce mondial.

\section{Conclusion}

67 Les transformations dans l'économie internationale ont conduit à la création de blocs économiques ou de zones économiques autour des principales économies développées. L' ALENA, par exemple, représente une série de défis aussi bien que des opportunités pour 
les pays de la Caraïbe. En effet, ce grand marché, centré autour des Etats-Unis, est une voie stratégique dynamiquement appropriée pour que la zone Caraïbe puisse réussir son insertion au Nouvel ordre économique mondial. Le marché nord-américain reste une destination non négligeable pour les exportations caribéennes, supérieur généralement, au marché intra-caribéen.

Pour réussir cette entreprise, les pays de la Caraïbe doivent restructurer leur économie afin de s'adapter aux nouvelles exigences des marchés globaux, notamment celui de l' ALENA. Il demeure cependant que la plupart des pays de la région ne remplissent pas encore les critères essentiels d'adhésion à l'ALENA. En outre, l'adhésion du Mexique au marché risque de mettre en cause les préférences commerciales tournées jusqu'alors vers les pays de la Caraïbe. A cela s'ajoute la nouvelle forme de dépendance économique des pays de la région vis-à-vis des politiques extérieures menées par des organismes internationaux tels que la Banque mondiale et le Fond monétaire international.

Les pays de la Caraïbe ont toujours essayé de répondre aux transformations subies par l'économie mondiale. Les différentes stratégies de développement économique mises en place dans la Caraïbe témoignent de cette démarche. Le succès relatif du modèle de développement de Porto Rico, l'Operation Bootstrap, par exemple, témoigne de cette capacité des économies de faire face aux demandes endogènes et exogènes. Et, bien que ces pays présentent des contraintes qui mettent en cause leur adhésion à l'ALENA, ils ont désormais des avantages comparatifs non négligeables qui favorisent leur insertion à ce marché : des variables technologiques, du savoir-faire, de l'efficacité dans les secteurs des services et de la manufacture de produits à haut et à moyen contenu technologique. De plus, il y a eu dans la zone à l'adaptation progressive d'innovation technologique. Or, c'est précisément l'innovation qui a constitué l'avantage comparatif qui a permis la montée graduelle des pays asiatiques dans les dernières décennies. Derrière le Japon, par exemple, se trouvent les pays asiatiques qui ont développé un système d'industrialisation général fondé sur le renouvellement permanent des avantages comparatifs.

Dans le nouvel ordre économique international, les variables de spécialisation et d'innovation occupent une position clé dans la dynamique des échanges internationaux. La spécialisation d'un pays n'est pas, toutefois, le résultat d'une adaptation passive à des tendances spontanées (comme dans le cas de l'Amérique Latine), elle doit être issue d'un effort continu afin d'utiliser au mieux ses atouts dans un environnement en constante évolution. Comme le souligne Gérard Lafay et Colette Herzog ${ }^{19}$, seule l'innovation peut créer des avantages comparatifs et il faut les renouveler progressivement, soit par le lancement de nouveaux produits, soit par l'adoption de processus de production plus efficaces. Pour cela, il faut que les entreprises mettent en place des stratégies en coordination avec l'Etat et la classe entrepreneuriale nationale; autrement dit c'est l'intégration des efforts des acteurs qui déterminent l'avenir du pays.

71 Il me semble que les pays de la Caraïbe ont déjà commencé à construire une voie permettant leur insertion au grand marché de l'ALENA. La tâche la plus difficile est de réussir une véritable intégration économique de la zone. Toutefois, l'histoire économique de ces pays a démontré que cette région est capable de faire face aux mutations de l'environnement économique mondial. Certains pays de la Caraïbe pourraient presque s'intégrer dans des circuits de production et de distribution plus compétitifs, avec une valeur ajoutée élevée puisqu'ils possèdent des avantages compétitifs axés sur la spécialisation et l'innovation progressive. 
Mais, il est important de savoir d'une part, si les entreprises attirées par des investissements dans la zone dirigent leurs flux d'investissement à cause de ces avantages comparatifs, sans tenir compte de l'avantage comparatif constitué par une main d'œuvre bon marché, et de l'autre, si les pays de la région sont disposés à mettre en place des politiques internes favorables comme, par exemple, celle d'augmenter le taux d'épargne national, d'investir dans l'éducation technologique, la recherche et le développement, ainsi que dans la rénovation des infrastructures.

\section{BIBLIOGRAPHIE}

APONTE GARCIA Maribel, « Caribbean and Latin American Economic Development Strategy: Theoretical Deconstruction within the regional Integration Context »,Postintegration Development in the Caribbean, Rio Piedras, Porto Rico, Social Science Research Center, 1995, pp. 18-74.

BENKO Georges et LIPIETZ Alain, Les régions qui gagnent, Paris, PUF, 1992.

CASTANER Juan, « La Liberalización del Comercio en el Hemisferio Occidental bajo el ALCH y la Económia de Puerto Rico », Ceteris Paribus, vol. 5, n 1, avril, 1995, pp. 59-81.

CELIMENE Fred et LACOUR Claude, L'intégration régionale des espaces, Paris, Economica, 1997. DEERE Carmen D. et MELENDEZ Edwin, « U.S. Trade Policy and Caribbean Economic Recovery: Sorting out the Contradictions ", Homines, vol. 17, n 1-2, juillet 1993-juin 1994, pp. 353-361.

HARKER Trevor, « Un Desarrollo Sostenido para el Caribe », Revista de la CEPAL, n 41, août, 1990, pp. 171-194.

KRUGMAN Paul, Peddling Prosperity: Economic sense and nonsense in the age of Diminished Expectation, N.Y., W.W. Norton \& Company, 1994.

LAFAY Gérard et HERZOG Colette, Commerce international, la fin des avantages acquis, Paris, Economica, 1989.

MUCCHIELLI Jean-Louis, Les firmes multinationales : mutations et nouvelles perspectives, Paris, Economica, 1985.

RAINELLI Michel, Le commerce international, Paris, La Découverte, 1992.

SERBIN Andrés, « El Caribe, Estados Unidos y América Latina en la Década del noventa : Ménage à trois ou partouze ?», Política Internacional, n² 25, janvier-mars, 1992, pp. 213-224.

WORRELL R. Delisle, « Economic Integration with Unequal Partners: the Caribbean and North America ", Working Papers, The Latin American Program of Washington Woodrow Wilson international Center for Scholars, $\mathrm{n}^{\circ}$ 205, décembre, 1993, pp. 1-42.

\section{ANNEXES}

Lexique 


\begin{tabular}{|l|l|}
\hline $\begin{array}{l}\text { Operation } \\
\text { Bootstrap }\end{array}$ & $\begin{array}{l}\text { modèle de développement économique mise en œuvre a Porto Rico à partir des } \\
\text { années 1940. Celui-ci est un modèle d'accumulation de capital basé sur les } \\
\text { investissements étrangers. }\end{array}$ \\
\hline Section 936 & $\begin{array}{l}\text { législation du Code des impôts fédéraux qui permet l'implantation à Porto Rico } \\
\text { des grandes entreprises multinationales grâce aux exonérations du paiement } \\
\text { d'impôts fédéraux sur tout le revenu produit par les activités industrielles et de } \\
\text { services des entreprises sur le territoire, ainsi que pour les dividendes rapatriés } \\
\text { vers la maison mère. }\end{array}$ \\
\hline $\begin{array}{l}\text { Toll-gate } \\
\text { tax }\end{array}$ & $\begin{array}{l}\text { impôt sur le revenu dérivé des investissements financiers des entreprises } \\
\text { installées à Porto Rico dans le cadre de la section 936. }\end{array}$ \\
\hline Twin-plants & $\begin{array}{l}\text { entreprises de production complémentaires ou des activités jumelées installées à } \\
\text { Porto Rico et dans les pays de la Caraïbe, en prenant en considération le capital, } \\
\text { la main-d'œuvre, ainsi que le facteur technologique. }\end{array}$ \\
\hline
\end{tabular}

\section{NOTES}

1. . Worrell, R. Delisle, « Economic Integration with Unequal Partners : the Carribbean and North America ", Working Papers, The Latin American Program of Washington Woodrow Wilson Center for Scholars, $\mathrm{n}^{\circ} 205$, décembre 1993, p. 5.

2. . Watson, Hilbourne A., "Globalization, New Regionalization, Restructuring, and NAFTA: Implications for the Signatories and the Caribbean ", Caribbean Studies, vol. 29, n 1, 1996, p. 38.

3. . Hillcoat G. et Quenan C., "L'intégration régionale dans les Caraïbes ", Cahiers des Amériques latines, $\mathrm{n}^{\circ} 12,1991$, pp. 139-164.

4. . Hillcoat G. et Quenan C., op. cit. Pour plus de détails voir aussi : Célimène Fred, «Vers de nouveaux modèles de développement dans la Caraïbe dans les années 90 ? ", dossier présenté pendant le Colloque International organisé par le CRPLC de l'Université des Antilles et de la Guyane, novembre 1994.

5. . Voir Quick Stephen, «The International Economy and the Caribbean: the 1990s and beyond »,Democracyin the Caribbean : political, economic ans social perspectives, Londres, The John Hopkins University Press, 1993. Mc Affe Kathy, Storm Signals, Boston, MA, South End Press, 1991.

6. . Voir Dietz James, Historia Economica de Puerto Rico, Rio Piedras, P.R., Huracan, 1991.

7. . Voir sur ce sujet, Gautier Mayoral Carmen, « The Puerto Rican Model and the CBI : a new form of south-south cooperation?", Caribbean Studies, vol. 23, n 1-2, 1990, pp. 10-12 ; Hillcoat G. et Quenan C, op. cit.

8. . Les technocrates du Département du Trésor des Etats-Unis proposaient la suppression de la Section 936 comme mécanisme pour conjurer le déficit fédéral puisqu'ils pourraient ainsi recueillir les revenus contributifs de ce flux de capital, pour autant que les entreprises ne fassent pas faillite. Alegria Ortega I., Lockwood W. et Rivera Ortiz A., «Las Relaciones Economicas de Puerto Rico en la Cuenca del Caribe : perspectivas de autonomia relativa o de subordinacion ", Puerto Rico en la Economia Politica del Caribe, Puerto Rico, Huracan, 1990.

9. . Ramos, Aaron G., " Porto Rico et les Etats-Unis : la crise du compromis territorial », in Justin Daniel, Les îles Caraibes, Paris, Karthala, 1996.

10. . Pour plus de détails voir: Eeuwen D. et Pizetty-van Eeuwen Y., "Intégrer ou séparer la question des indépendances, enjeu politique dans la Caraïbe?", La transformation de l'Etat en Amérique Latine, Paris, Karthala, 1995. Delgado J. et Sanchez O.M., et Ceara-Hatton M., «Las 
economias caribenas en la década de los ochenta ", revue Revista de Ciencias Sociales, vol. 27, n - 3-4, 1988, pp. 83-115.

11. . Pantojas E. et Dietz J., « Neoliberal Policies and Caribbean Development: from de CBI to NAFTA », revue21 ${ }^{\text {st }}$ Century policy review, vol. 2, n 1-2, printemps, 1994.

12. . Watson Hilbourne A., "Globalization, New Regionalization, Restructuring, and NAFTA : Implications for the Signatories and the Caribbean », Caribbean Studies, vol. 29, n 1, 1996, pp. 5-48.

13. . Hatem Fabrice, Les multinationales en l'an 2000, Paris, Economica, 1995.

14. . Puerto Rico Planning Board, Informe Económico al Gobernador, Porto Rico, PRPB, 1995.

15. . Government Development Bank for Puerto Rico, Annual Report, Porto Rico, GDB, 1996.

16. . Crusol, J. et Vellas, F., Le tourisme et la Caraïbe, Paris, L'Harmattan, 1996.

17. Government Development Bank for Puerto Rico, Annual Report, Porto Rico, GDB, 1996.

18. Mucchielli, Les firmes multinationales: mutations et nouvelles perspectives, Paris, Economica, 1985, p. 22.

19. . Gérard Lafay et Colette Herzog, Commerce international, la fin des avantages acquis, Paris, Economica, 1989.

\section{RÉSUMÉS}

Les nouvelles tendances de l'économie internationale donnent lieu à la formation de grandes régions ou zones économiques autour des principales économies développées. En Amérique, l'Accord de libre échange nord-américain (ALENA) créent désormais de nouveaux défis et des contraintes pour les économies de la Caraïbe, notamment pour celle de Porto Rico. En effet, pour ces économies les principales contraintes face à l'ALENA sont l'adhésion du Mexique et, la nouvelle forme de dépendance de ces pays vis-à-vis des politiques exogènes gérées particulièrement par des organismes internationaux (BM et FMI). En prenant le cas de Porto Rico, on constate que la Caraïbe a des avantages comparatifs qui peuvent permettre, dans un avenir proche, son insertion au grand marché de l'ALENA. Il s'agit d'une main-d'œuvre qualifiée et d'une dynamique de spécialisation et d'innovation progressive à l'intérieur du processus productif dans certains secteurs clés (services, manufacture de produits à moyen et à haut contenu technologique).

The new trends of inter-national economy are geared towards allowing the formation of great economies or economic regions around the main or principal economies already developed and established. In America, the NAFTA represents new challenges and tensions for the Caribbean economies like that of Puerto Rico. The main worries of these economies are the possibilities of including Mexico in the NAFTA, mainly because of the corporate reliefs that the Caribbean region enjoyed are going to disappear, and the new forms of dependency of these countries facing with the external policies managed by the international bodies such as (WB) and (IMF). Using Puerto Rico as an exemple there is proof that the Caribbean enjoys comparative advantages that can in a very near future allow this region its entrance to the great trade market of NAFTA. These avantages are, a qualified work force and a dynamics of specialization and continual innovation in certain sectors of the production process (as in services and the manufacture of advanced and higher technology). 
INDEX

Index géographique : Antilles, Porto-Rico

Mots-clés : Alena, économie, fmi, Opération Bootstraps, Section 936

Keywords : economie, ifm, nAfta, Operation Bootstraps, Section 936

\section{AUTEUR}

KAREN L. ORENGO-SERRA

Doctorante en sciences économiques

Institut des Hautes Etudes de l'Amérique Latine 